Supplement of Earth Syst. Sci. Data, 13, 213-229, 2021

https://doi.org/10.5194/essd-13-213-2021-supplement

(C) Author(s) 2021. This work is distributed under

the Creative Commons Attribution 4.0 License.

(c) (1)

Supplement of

\title{
Dataset of Georeferenced Dams in South America (DDSA)
}

Bolivar Paredes-Beltran et al.

Correspondence to: Bolivar Paredes-Beltran (be.paredes@alumnos.upm.es, be.paredes@uta.edu.ec)

The copyright of individual parts of the supplement might differ from the CC BY 4.0 License. 
SUPPLEMENTARY TABLE S1

FUTURE DAMS IN SOUTH AMERICA

\begin{tabular}{|c|c|c|c|}
\hline COUNTRY & NAME & PHASE & SOURCE \\
\hline Argentina & Brazo Aña Cua & Projected & FHReD, 2015 \\
\hline Argentina & Cerro Rayoso & Projected & FHReD, 2015 \\
\hline Argentina & Chihuido 1 & Projected & FHReD, 2015 \\
\hline Argentina & Condor Cliff & In Construction & FHReD, 2015 \\
\hline Argentina & Corpus Christi & Projected & FHReD, 2015 \\
\hline Argentina & El Tambolar & In Construction & FHReD, 2015 \\
\hline Argentina & Garabi & Projected & FHReD, 2015 \\
\hline Argentina & Itacora-Itati & Projected & FHReD, 2015 \\
\hline Argentina & La Barrancosa & In Construction & FHReD, 2015 \\
\hline Argentina & La Invernada & Projected & FHReD, 2015 \\
\hline Argentina & Los Blancos I & Projected & FHReD, 2015 \\
\hline Argentina & Los Blancos II & Projected & FHReD, 2015 \\
\hline Argentina & Michihuao & Projected & FHReD, 2015 \\
\hline Argentina & Panambi & Projected & FHReD, 2015 \\
\hline Argentina & Piedra del Aguila (expansion) & Projected & FHReD, 2015 \\
\hline Argentina & Portezuelo del Viento & Projected & FHReD, 2015 \\
\hline Argentina & Potrero del Clavillo & Projected & FHReD, 2015 \\
\hline Argentina & Yacyreta (expansion) & Projected & FHReD, 2015 \\
\hline Bolivia & Ambrosia & Projected & FHReD, 2015; Almeida et al., 2019 \\
\hline Bolivia & Angosto Chepete & Projected & FHReD, 2015; Almeida et al., 2019 \\
\hline Bolivia & Angosto del Bala & Projected & FHReD, 2015; Almeida et al., 2019 \\
\hline Bolivia & Bacata & Projected & FHReD, 2015; Almeida et al., 2019 \\
\hline Bolivia & Banda Azul & Projected & FHReD, 2015; Almeida et al., 2019 \\
\hline Bolivia & Cañahuecal & Projected & FHReD, 2015; Almeida et al., 2019 \\
\hline Bolivia & Cachuela Esperanza & Projected & FHReD, 2015; Almeida et al., 2019 \\
\hline Bolivia & Carrizal & Projected & FHReD, 2015; Almeida et al., 2019 \\
\hline Bolivia & Guajará-Mirim & Projected & FHReD, 2015; Almeida et al., 2019 \\
\hline Bolivia & Icona & Projected & FHReD, 2015; Almeida et al., 2019 \\
\hline Bolivia & Ivirizu & In Construction & FHReD, 2015; Almeida et al., 2019 \\
\hline Bolivia & Jatun Pampa & Projected & FHReD, 2015; Almeida et al., 2019 \\
\hline Bolivia & La Pesca & Projected & FHReD, 2015; Almeida et al., 2019 \\
\hline Bolivia & Las Juntas & Projected & FHReD, 2015; Almeida et al., 2019 \\
\hline Bolivia & Ocampo & Projected & FHReD, 2015; Almeida et al., 2019 \\
\hline Bolivia & Pachalaca & Projected & FHReD, 2015; Almeida et al., 2019 \\
\hline Bolivia & Palillada & In Construction & FHReD, 2015; Almeida et al., 2019 \\
\hline Bolivia & Peña Blanca & Projected & FHReD, 2015; Almeida et al., 2019 \\
\hline Bolivia & Rio Grande - Rositas & Projected & FHReD, 2015; Almeida et al., 2019 \\
\hline Bolivia & Rio Madera & Projected & FHReD, 2015; Almeida et al., 2019 \\
\hline Bolivia & Rositas & Projected & FHReD, 2015 \\
\hline Bolivia & Sehuencas & Projected & FHReD, 2015; Almeida et al., 2019 \\
\hline Bolivia & Umapalca & In Construction & FHReD, 2015; Almeida et al., 2019 \\
\hline Bolivia & Seripona & Projected & FHReD, 2015; Almeida et al., 2019 \\
\hline Brazil & 40 Ilhas & Projected & FHReD, 2015; Almeida et al., 2019 \\
\hline Brazil & A1e6 & Projected & FHReD, 2015; Almeida et al., 2019 \\
\hline Brazil & A1e8 & Projected & FHReD, 2015; Almeida et al., 2019 \\
\hline Brazil & A1e9 & Projected & FHReD, 2015; Almeida et al., 2019 \\
\hline Brazil & A2e11 & Projected & FHReD, 2015; Almeida et al., 2019 \\
\hline
\end{tabular}




\begin{tabular}{|c|c|c|c|}
\hline COUNTRY & NAME & PHASE & SOURCE \\
\hline Brazil & A2e12 & Projected & FHReD, 2015; Almeida et al., 2019 \\
\hline Brazil & A2e13 & Projected & FHReD, 2015; Almeida et al., 2019 \\
\hline Brazil & A2e14 & Projected & FHReD, 2015; Almeida et al., 2019 \\
\hline Brazil & A2e15 & Projected & FHReD, 2015; Almeida et al., 2019 \\
\hline Brazil & A2e16 & Projected & FHReD, 2015; Almeida et al., 2019 \\
\hline Brazil & A2e17 & Projected & FHReD, 2015; Almeida et al., 2019 \\
\hline Brazil & A2e18 & Projected & FHReD, 2015; Almeida et al., 2019 \\
\hline Brazil & A2e4 & Projected & FHReD, 2015; Almeida et al., 2019 \\
\hline Brazil & A2e5 & Projected & FHReD, 2015; Almeida et al., 2019 \\
\hline Brazil & A2e6 & Projected & FHReD, 2015; Almeida et al., 2019 \\
\hline Brazil & A2e7 & Projected & FHReD, 2015; Almeida et al., 2019 \\
\hline Brazil & A2e8 & Projected & FHReD, 2015; Almeida et al., 2019 \\
\hline Brazil & A2e9 & Projected & FHReD, 2015; Almeida et al., 2019 \\
\hline Brazil & A38pa100 & Projected & FHReD, 2015; Almeida et al., 2019 \\
\hline Brazil & A41pa008 & Projected & FHReD, 2015; Almeida et al., 2019 \\
\hline Brazil & A5 L10 & Projected & FHReD, 2015; Almeida et al., 2019 \\
\hline Brazil & A8b & Projected & FHReD, 2015; Almeida et al., 2019 \\
\hline Brazil & Acaipe & Projected & FHReD, 2015; Almeida et al., 2019 \\
\hline Brazil & Açungui 2E & Projected & FHReD, 2015, ANEEL, 2020 \\
\hline Brazil & Açungui $2 \mathrm{~F}$ & Projected & FHReD, 2015, ANEEL, 2020 \\
\hline Brazil & Agua Boa & Projected & FHReD, 2015, ANEEL, 2020; Almeida et al., 2019 \\
\hline Brazil & Águas da Serra & Projected & FHReD, 2015, ANEEL, 2020 \\
\hline Brazil & Aguas Lindas & Projected & FHReD, 2015, ANEEL, 2020; Almeida et al., 2019 \\
\hline Brazil & Alcocaba & Projected & FHReD, 2015, ANEEL, 2020; Almeida et al., 2019 \\
\hline Brazil & Alto Alegre & Projected & FHReD, 2015, ANEEL, 2020 \\
\hline Brazil & Alto Garcia & Projected & FHReD, 2015, ANEEL, 2020 \\
\hline Brazil & Alto Guaporé 2 & Projected & FHReD, 2015, ANEEL, 2020 \\
\hline Brazil & Âmbar & In Construction & FHReD, 2015, ANEEL, 2020 \\
\hline Brazil & Andorinha & Projected & FHReD, 2015, ANEEL, 2020; Almeida et al., 2019 \\
\hline Brazil & Antônio Dias (Antiga Água Limpa) & Projected & FHReD, 2015, ANEEL, 2020 \\
\hline Brazil & Apertadinho & In Construction & FHReD, 2015, ANEEL, 2020 \\
\hline Brazil & Apiaka Kayabi PEX-093 & Projected & FHReD, 2015, ANEEL, 2020; Almeida et al., 2019 \\
\hline Brazil & Armando Ribeiro & In Construction & FHReD, 2015, ANEEL, 2020 \\
\hline Brazil & Arn 026 & Projected & FHReD, 2015, ANEEL, 2020; Almeida et al., 2019 \\
\hline Brazil & Arrozeira Meyer & Projected & FHReD, 2015, ANEEL, 2020 \\
\hline Brazil & Aruana & Projected & FHReD, 2015, ANEEL, 2020; Almeida et al., 2019 \\
\hline Brazil & Bacupari & Projected & FHReD, 2015, ANEEL, 2020; Almeida et al., 2019 \\
\hline Brazil & Bacuri & Projected & FHReD, 2015, ANEEL, 2020; Almeida et al., 2019 \\
\hline Brazil & Bananal & Projected & FHReD, 2015, ANEEL, 2020; Almeida et al., 2019 \\
\hline Brazil & Barra Do Claro & Projected & FHReD, 2015, ANEEL, 2020; Almeida et al., 2019 \\
\hline Brazil & Barra Do Gaviao & Projected & FHReD, 2015, ANEEL, 2020; Almeida et al., 2019 \\
\hline Brazil & Barreiros & Projected & FHReD, 2015, ANEEL, 2020; Almeida et al., 2019 \\
\hline Brazil & Bela Vista & Projected & FHReD, 2015, ANEEL, 2020 \\
\hline Brazil & Bela Vista & In Construction & FHReD, 2015, ANEEL, 2020 \\
\hline Brazil & Bem Querer & Projected & FHReD, 2015, ANEEL, 2020; Almeida et al., 2019 \\
\hline Brazil & Berimbau & Projected & FHReD, 2015, ANEEL, 2020; Almeida et al., 2019 \\
\hline Brazil & Boa Vista & Projected & FHReD, 2015, ANEEL, 2020 \\
\hline Brazil & Bom Jesus & Projected & FHReD, 2015, ANEEL, 2020 \\
\hline Brazil & Braco Leste & Projected & FHReD, 2015, ANEEL, 2020; Almeida et al., 2019 \\
\hline
\end{tabular}




\begin{tabular}{|c|c|c|c|}
\hline COUNTRY & NAME & PHASE & SOURCE \\
\hline Brazil & Braco Sul & Projected & FHReD, 2015, ANEEL, 2020; Almeida et al., 2019 \\
\hline Brazil & Brejaúba & Projected & FHReD, 2015, ANEEL, 2020 \\
\hline Brazil & Buriti & Projected & FHReD, 2015, ANEEL, 2020; Almeida et al., 2019 \\
\hline Brazil & Buritizal 1 & Projected & FHReD, 2015, ANEEL, 2020; Almeida et al., 2019 \\
\hline Brazil & Buritizal 2 & Projected & FHReD, 2015, ANEEL, 2020; Almeida et al., 2019 \\
\hline Brazil & Buritizal 3 & Projected & FHReD, 2015, ANEEL, 2020; Almeida et al., 2019 \\
\hline Brazil & Cabeca de Boi & Projected & FHReD, 2015, ANEEL, 2020; Almeida et al., 2019 \\
\hline Brazil & Cabuí & Projected & FHReD, 2015, ANEEL, 2020 \\
\hline Brazil & Cachimbo & Projected & FHReD, 2015, ANEEL, 2020; Almeida et al., 2019 \\
\hline Brazil & Cachoeira Cinco Veados & In Construction & FHReD, 2015, ANEEL, 2020 \\
\hline Brazil & Cachoeira do Miné & Projected & FHReD, 2015, ANEEL, 2020 \\
\hline Brazil & Cachoeira Agua Preta & Projected & FHReD, 2015, ANEEL, 2020; Almeida et al., 2019 \\
\hline Brazil & Cachoeira Cachimbo Alto & Projected & FHReD, 2015, ANEEL, 2020; Almeida et al., 2019 \\
\hline Brazil & Cachoeira Caracol & Projected & FHReD, 2015, ANEEL, 2020; Almeida et al., 2019 \\
\hline Brazil & Cachoeira do Cambara & Projected & FHReD, 2015, ANEEL, 2020; Almeida et al., 2019 \\
\hline Brazil & Cachoeira do Codo & Projected & FHReD, 2015, ANEEL, 2020; Almeida et al., 2019 \\
\hline Brazil & Cachoeira do Ebrio & Projected & FHReD, 2015, ANEEL, 2020; Almeida et al., 2019 \\
\hline Brazil & Cachoeira do Meio & Projected & FHReD, 2015, ANEEL, 2020; Almeida et al., 2019 \\
\hline Brazil & Cachoeira Formosa & Projected & FHReD, 2015, ANEEL, 2020; Almeida et al., 2019 \\
\hline Brazil & Cachoeira Fortaleza & Projected & FHReD, 2015, ANEEL, 2020; Almeida et al., 2019 \\
\hline Brazil & Cachoeira Galinha & Projected & FHReD, 2015, ANEEL, 2020; Almeida et al., 2019 \\
\hline Brazil & Cachoeira Sao Jose & Projected & FHReD, 2015, ANEEL, 2020; Almeida et al., 2019 \\
\hline Brazil & Cachoeira Do Cai & Projected & FHReD, 2015, ANEEL, 2020; Almeida et al., 2019 \\
\hline Brazil & Cachoeira Dos Patos & Projected & FHReD, 2015, ANEEL, 2020; Almeida et al., 2019 \\
\hline Brazil & Cachoeirao & Projected & FHReD, 2015, ANEEL, 2020; Almeida et al., 2019 \\
\hline Brazil & Caldeirões & Projected & FHReD, 2015, ANEEL, 2020 \\
\hline Brazil & Camberra 2 & Projected & FHReD, 2015, ANEEL, 2020; Almeida et al., 2019 \\
\hline Brazil & Campo Belo & Projected & FHReD, 2015, ANEEL, 2020 \\
\hline Brazil & Campos de Julio & Projected & FHReD, 2015, ANEEL, 2020; Almeida et al., 2019 \\
\hline Brazil & Candeia & Projected & FHReD, 2015, ANEEL, 2020; Almeida et al., 2019 \\
\hline Brazil & Canoa Quebrada & Projected & FHReD, 2015, ANEEL, 2020; Almeida et al., 2019 \\
\hline Brazil & Carnauba & Projected & FHReD, 2015, ANEEL, 2020; Almeida et al., 2019 \\
\hline Brazil & Cascata & Projected & FHReD, 2015, ANEEL, 2020 \\
\hline Brazil & Castanhão & In Construction & FHReD, 2015, ANEEL, 2020 \\
\hline Brazil & Castanheira 2 & Projected & FHReD, 2015, ANEEL, 2020; Almeida et al., 2019 \\
\hline Brazil & Castanheira AR N-120 & Projected & FHReD, 2015, ANEEL, 2020; Almeida et al., 2019 \\
\hline Brazil & Cavernoso III & Projected & FHReD, 2015, ANEEL, 2020 \\
\hline Brazil & Cavernoso IV & Projected & FHReD, 2015, ANEEL, 2020 \\
\hline Brazil & Cedro & Projected & FHReD, 2015, ANEEL, 2020; Almeida et al., 2019 \\
\hline Brazil & Cesar Filho & Projected & FHReD, 2015, ANEEL, 2020; Almeida et al., 2019 \\
\hline Brazil & Cerquinha II & Projected & FHReD, 2015, ANEEL, 2020 \\
\hline Brazil & Cerquinha III & Projected & FHReD, 2015, ANEEL, 2020 \\
\hline Brazil & Chacorao & Projected & FHReD, 2015, ANEEL, 2020; Almeida et al., 2019 \\
\hline Brazil & Cianorte & Projected & FHReD, 2015, ANEEL, 2020 \\
\hline Brazil & Cinta Larga & Projected & FHReD, 2015, ANEEL, 2020; Almeida et al., 2019 \\
\hline Brazil & Cozumel 2 & Projected & FHReD, 2015, ANEEL, 2020; Almeida et al., 2019 \\
\hline Brazil & Cristalina & Projected & FHReD, 2015, ANEEL, 2020; Almeida et al., 2019 \\
\hline Brazil & Diauarum & Projected & FHReD, 2015, ANEEL, 2020; Almeida et al., 2019 \\
\hline Brazil & Distancia & Projected & FHReD, 2015, ANEEL, 2020; Almeida et al., 2019 \\
\hline
\end{tabular}




\begin{tabular}{|c|c|c|c|}
\hline COUNTRY & NAME & PHASE & SOURCE \\
\hline Brazil & Divisa & Projected & FHReD, 2015, ANEEL, 2020; Almeida et al., 2019 \\
\hline Brazil & Erikpatsa JRN-530 & Projected & FHReD, 2015, ANEEL, 2020; Almeida et al., 2019 \\
\hline Brazil & Escondido JRN-277 & Projected & FHReD, 2015, ANEEL, 2020; Almeida et al., 2019 \\
\hline Brazil & Esperanca & Projected & FHReD, 2015, ANEEL, 2020; Almeida et al., 2019 \\
\hline Brazil & Faveiro & Projected & FHReD, 2015, ANEEL, 2020; Almeida et al., 2019 \\
\hline Brazil & Fe Esperanca & Projected & FHReD, 2015, ANEEL, 2020; Almeida et al., 2019 \\
\hline Brazil & Formiga & Projected & FHReD, 2015, ANEEL, 2020; Almeida et al., 2019 \\
\hline Brazil & Foz Formiga Baixo JUI-029b & Projected & FHReD, 2015, ANEEL, 2020; Almeida et al., 2019 \\
\hline Brazil & Foz Sacre PPG-147 & Projected & FHReD, 2015, ANEEL, 2020; Almeida et al., 2019 \\
\hline Brazil & Foz Do Apiacas & Projected & FHReD, 2015, ANEEL, 2020; Almeida et al., 2019 \\
\hline Brazil & Frieira & Projected & FHReD, 2015, ANEEL, 2020; Almeida et al., 2019 \\
\hline Brazil & Clairto Zonta & Projected & FHReD, 2015, ANEEL, 2020 \\
\hline Brazil & Cobre Km 19 & Projected & FHReD, 2015, ANEEL, 2020 \\
\hline Brazil & Comodoro & Projected & FHReD, 2015, ANEEL, 2020 \\
\hline Brazil & Confluência & Projected & FHReD, 2015, ANEEL, 2020 \\
\hline Brazil & Córrego Fundo & Projected & FHReD, 2015, ANEEL, 2020 \\
\hline Brazil & Couro do Cervo & Projected & FHReD, 2015, ANEEL, 2020 \\
\hline Brazil & Dias & Projected & FHReD, 2015, ANEEL, 2020 \\
\hline Brazil & Dois Saltos & In Construction & FHReD, 2015, ANEEL, 2020 \\
\hline Brazil & Emas Nova & Projected & FHReD, 2015, ANEEL, 2020 \\
\hline Brazil & Estação Indaial & Projected & FHReD, 2015, ANEEL, 2020 \\
\hline Brazil & Estivadinho 3 & Projected & FHReD, 2015, ANEEL, 2020 \\
\hline Brazil & Fazenda do Salto & In Construction & FHReD, 2015, ANEEL, 2020 \\
\hline Brazil & Fazenda Santana & Projected & FHReD, 2015, ANEEL, 2020 \\
\hline Brazil & Forquilha & In Construction & FHReD, 2015, ANEEL, 2020 \\
\hline Brazil & Forquilha IV & In Construction & FHReD, 2015, ANEEL, 2020 \\
\hline Brazil & Fortaleza & Projected & FHReD, 2015, ANEEL, 2020 \\
\hline Brazil & Foz da Anta & Projected & FHReD, 2015, ANEEL, 2020 \\
\hline Brazil & Foz do Cedro (Antiga A1E8) & Projected & FHReD, 2015, ANEEL, 2020 \\
\hline Brazil & Foz do Estrela & In Construction & FHReD, 2015, ANEEL, 2020 \\
\hline Brazil & Foz do Santana & Projected & FHReD, 2015, ANEEL, 2020 \\
\hline Brazil & Galera & Projected & FHReD, 2015, ANEEL, 2020; Almeida et al., 2019 \\
\hline Brazil & Garca & Projected & FHReD, 2015, ANEEL, 2020; Almeida et al., 2019 \\
\hline Brazil & Gaucha Do Norte & Projected & FHReD, 2015, ANEEL, 2020; Almeida et al., 2019 \\
\hline Brazil & Girassol & Projected & FHReD, 2015, ANEEL, 2020; Almeida et al., 2019 \\
\hline Brazil & Guapuruvu & Projected & FHReD, 2015, ANEEL, 2020; Almeida et al., 2019 \\
\hline Brazil & Iara & Projected & FHReD, 2015, ANEEL, 2020; Almeida et al., 2019 \\
\hline Brazil & Illha Sao Pedro & Projected & FHReD, 2015, ANEEL, 2020; Almeida et al., 2019 \\
\hline Brazil & Ilha Tres Quedas & Projected & FHReD, 2015, ANEEL, 2020; Almeida et al., 2019 \\
\hline Brazil & Illhotas & Projected & FHReD, 2015, ANEEL, 2020; Almeida et al., 2019 \\
\hline Brazil & Inferninho & Projected & FHReD, 2015, ANEEL, 2020; Almeida et al., 2019 \\
\hline Brazil & Ipiranga & Projected & FHReD, 2015, ANEEL, 2020; Almeida et al., 2019 \\
\hline Brazil & Itacare & Projected & FHReD, 2015, ANEEL, 2020; Almeida et al., 2019 \\
\hline Brazil & Jaborandi & Projected & FHReD, 2015, ANEEL, 2020; Almeida et al., 2019 \\
\hline Brazil & Jaboticabal & Projected & FHReD, 2015, ANEEL, 2020; Almeida et al., 2019 \\
\hline Brazil & Jaburu & Projected & FHReD, 2015, ANEEL, 2020; Almeida et al., 2019 \\
\hline Brazil & Jamanxim & Projected & FHReD, 2015, ANEEL, 2020; Almeida et al., 2019 \\
\hline Brazil & Jardim de Ouro & Projected & FHReD, 2015, ANEEL, 2020; Almeida et al., 2019 \\
\hline Brazil & Jatoba & Projected & FHReD, 2015, ANEEL, 2020; Almeida et al., 2019 \\
\hline
\end{tabular}




\begin{tabular}{|c|c|c|c|}
\hline COUNTRY & NAME & PHASE & SOURCE \\
\hline Brazil & Jeriva & Projected & FHReD, 2015, ANEEL, 2020; Almeida et al., 2019 \\
\hline Brazil & Gameleira & Projected & FHReD, 2015, ANEEL, 2020 \\
\hline Brazil & Guaraú & Projected & FHReD, 2015, ANEEL, 2020 \\
\hline Brazil & Ibituruna & Projected & FHReD, 2015, ANEEL, 2020 \\
\hline Brazil & Invernadinha & In Construction & FHReD, 2015, ANEEL, 2020 \\
\hline Brazil & Itaocara I & Projected & FHReD, 2015, ANEEL, 2020 \\
\hline Brazil & Itiquira III & Projected & FHReD, 2015, ANEEL, 2020 \\
\hline Brazil & Jaspe & Projected & FHReD, 2015, ANEEL, 2020 \\
\hline Brazil & Jesuíta & Projected & FHReD, 2015, ANEEL, 2020; Almeida et al., 2019 \\
\hline Brazil & Ji Parana & Projected & FHReD, 2015, ANEEL, 2020; Almeida et al., 2019 \\
\hline Brazil & Joacaba & Projected & FHReD, 2015, ANEEL, 2020; Almeida et al., 2019 \\
\hline Brazil & Jrn 577 & Projected & FHReD, 2015, ANEEL, 2020; Almeida et al., 2019 \\
\hline Brazil & Jrn 720 & Projected & FHReD, 2015, ANEEL, 2020; Almeida et al., 2019 \\
\hline Brazil & Jui 048 & Projected & FHReD, 2015, ANEEL, 2020; Almeida et al., 2019 \\
\hline Brazil & Jui 117 & Projected & FHReD, 2015, ANEEL, 2020; Almeida et al., 2019 \\
\hline Brazil & Juina 1 & Projected & FHReD, 2015, ANEEL, 2020; Almeida et al., 2019 \\
\hline Brazil & Juina 2 & Projected & FHReD, 2015, ANEEL, 2020; Almeida et al., 2019 \\
\hline Brazil & Juina 3 & Projected & FHReD, 2015, ANEEL, 2020; Almeida et al., 2019 \\
\hline Brazil & Juruena & Projected & FHReD, 2015, ANEEL, 2020; Almeida et al., 2019 \\
\hline Brazil & Jurureba & Projected & FHReD, 2015, ANEEL, 2020; Almeida et al., 2019 \\
\hline Brazil & Jutuarama & Projected & FHReD, 2015, ANEEL, 2020; Almeida et al., 2019 \\
\hline Brazil & Kabiara & Projected & FHReD, 2015, ANEEL, 2020; Almeida et al., 2019 \\
\hline Brazil & Kona & Projected & FHReD, 2015, ANEEL, 2020; Almeida et al., 2019 \\
\hline Brazil & Lagoa Rasa & Projected & FHReD, 2015, ANEEL, 2020; Almeida et al., 2019 \\
\hline Brazil & Macauba & Projected & FHReD, 2015, ANEEL, 2020; Almeida et al., 2019 \\
\hline Brazil & Juba IV & Projected & FHReD, 2015, ANEEL, 2020 \\
\hline Brazil & Jubinha II & Projected & FHReD, 2015, ANEEL, 2020 \\
\hline Brazil & Jubinha III & Projected & FHReD, 2015, ANEEL, 2020 \\
\hline Brazil & Lacerdópolis & Projected & FHReD, 2015, ANEEL, 2020 \\
\hline Brazil & Linha Aparecida & Projected & FHReD, 2015, ANEEL, 2020 \\
\hline Brazil & Linha Jacinto & Projected & FHReD, 2015, ANEEL, 2020 \\
\hline Brazil & Linha Rica & In Construction & FHReD, 2015, ANEEL, 2020 \\
\hline Brazil & Lucia Cherobim & Projected & FHReD, 2015, ANEEL, 2020 \\
\hline Brazil & Macacos & In Construction & FHReD, 2015, ANEEL, 2020 \\
\hline Brazil & Machadinho I & Projected & FHReD, 2015, ANEEL, 2020; Almeida et al., 2019 \\
\hline Brazil & Magessi & Projected & FHReD, 2015, ANEEL, 2020; Almeida et al., 2019 \\
\hline Brazil & Manaca & Projected & FHReD, 2015, ANEEL, 2020; Almeida et al., 2019 \\
\hline Brazil & Mangabeira & Projected & FHReD, 2015, ANEEL, 2020; Almeida et al., 2019 \\
\hline Brazil & Mangaratiba & Projected & FHReD, 2015, ANEEL, 2020; Almeida et al., 2019 \\
\hline Brazil & Martelo & Projected & FHReD, 2015, ANEEL, 2020; Almeida et al., 2019 \\
\hline Brazil & Mangueira de Pedra & Projected & FHReD, 2015, ANEEL, 2020 \\
\hline Brazil & Mantovilis & In Construction & FHReD, 2015, ANEEL, 2020 \\
\hline Brazil & Manuel Alves & In Construction & FHReD, 2015, ANEEL, 2020 \\
\hline Brazil & Matrinchã & Projected & FHReD, 2015, ANEEL, 2020; Almeida et al., 2019 \\
\hline Brazil & Membeca 1 & Projected & FHReD, 2015, ANEEL, 2020; Almeida et al., 2019 \\
\hline Brazil & Membeca 2 & Projected & FHReD, 2015, ANEEL, 2020; Almeida et al., 2019 \\
\hline Brazil & Membeca 4 & Projected & FHReD, 2015, ANEEL, 2020; Almeida et al., 2019 \\
\hline Brazil & Membeca 5 & Projected & FHReD, 2015, ANEEL, 2020; Almeida et al., 2019 \\
\hline Brazil & Membeca 6 & Projected & FHReD, 2015, ANEEL, 2020; Almeida et al., 2019 \\
\hline
\end{tabular}




\begin{tabular}{|c|c|c|c|}
\hline COUNTRY & NAME & PHASE & SOURCE \\
\hline Brazil & Membeca 8 & Projected & FHReD, 2015, ANEEL, 2020; Almeida et al., 2019 \\
\hline Brazil & Membeca 9 & Projected & FHReD, 2015, ANEEL, 2020; Almeida et al., 2019 \\
\hline Brazil & Minacu & Projected & FHReD, 2015, ANEEL, 2020; Almeida et al., 2019 \\
\hline Brazil & Miriti & Projected & FHReD, 2015, ANEEL, 2020; Almeida et al., 2019 \\
\hline Brazil & Mocoto & Projected & FHReD, 2015, ANEEL, 2020; Almeida et al., 2019 \\
\hline Brazil & Mogno & Projected & FHReD, 2015, ANEEL, 2020; Almeida et al., 2019 \\
\hline Brazil & Mu2 & Projected & FHReD, 2015, ANEEL, 2020; Almeida et al., 2019 \\
\hline Brazil & Mu3 & Projected & FHReD, 2015, ANEEL, 2020; Almeida et al., 2019 \\
\hline Brazil & Mutamba & Projected & FHReD, 2015, ANEEL, 2020; Almeida et al., 2019 \\
\hline Brazil & Nambikwara JUI-008 & Projected & FHReD, 2015, ANEEL, 2020; Almeida et al., 2019 \\
\hline Brazil & Nordeste & Projected & FHReD, 2015, ANEEL, 2020; Almeida et al., 2019 \\
\hline Brazil & Nossa Senhora Gracas 1 & Projected & FHReD, 2015, ANEEL, 2020; Almeida et al., 2019 \\
\hline Brazil & Melo Viana & Projected & FHReD, 2015, ANEEL, 2020 \\
\hline Brazil & Monjolo & Projected & FHReD, 2015, ANEEL, 2020 \\
\hline Brazil & Monte Cuco & Projected & FHReD, 2015, ANEEL, 2020 \\
\hline Brazil & Muçungo & Projected & FHReD, 2015, ANEEL, 2020 \\
\hline Brazil & Mutum I & Projected & FHReD, 2015, ANEEL, 2020 \\
\hline Brazil & Nhandu & In Construction & FHReD, 2015, ANEEL, 2020 \\
\hline Brazil & Nova Guaporé & In Construction & FHReD, 2015, ANEEL, 2020; Almeida et al., 2019 \\
\hline Brazil & Nova Mutum & Projected & FHReD, 2015, ANEEL, 2020; Almeida et al., 2019 \\
\hline Brazil & Paiagua & Projected & FHReD, 2015, ANEEL, 2020; Almeida et al., 2019 \\
\hline Brazil & Pancada Grande & Projected & FHReD, 2015, ANEEL, 2020; Almeida et al., 2019 \\
\hline Brazil & Paranatinga 1 & Projected & FHReD, 2015, ANEEL, 2020; Almeida et al., 2019 \\
\hline Brazil & Paredao A & Projected & FHReD, 2015, ANEEL, 2020; Almeida et al., 2019 \\
\hline Brazil & Paredao M1 & Projected & FHReD, 2015, ANEEL, 2020; Almeida et al., 2019 \\
\hline Brazil & Patos & Projected & FHReD, 2015, ANEEL, 2020; Almeida et al., 2019 \\
\hline Brazil & Pch J3 & Projected & FHReD, 2015, ANEEL, 2020; Almeida et al., 2019 \\
\hline Brazil & Pch J4 & Projected & FHReD, 2015, ANEEL, 2020; Almeida et al., 2019 \\
\hline Brazil & Pedra Branca & Projected & FHReD, 2015, ANEEL, 2020; Almeida et al., 2019 \\
\hline Brazil & Perdidos & Projected & FHReD, 2015, ANEEL, 2020; Almeida et al., 2019 \\
\hline Brazil & Pescara 2 & Projected & FHReD, 2015, ANEEL, 2020; Almeida et al., 2019 \\
\hline Brazil & Pitombeira & Projected & FHReD, 2015, ANEEL, 2020; Almeida et al., 2019 \\
\hline Brazil & Ppg 115 & Projected & FHReD, 2015, ANEEL, 2020; Almeida et al., 2019 \\
\hline Brazil & Prainha & Projected & FHReD, 2015, ANEEL, 2020; Almeida et al., 2019 \\
\hline Brazil & Precipicio & Projected & FHReD, 2015, ANEEL, 2020; Almeida et al., 2019 \\
\hline Brazil & Pucon 2 & Projected & FHReD, 2015, ANEEL, 2020; Almeida et al., 2019 \\
\hline Brazil & Quebra Remo & Projected & FHReD, 2015, ANEEL, 2020; Almeida et al., 2019 \\
\hline Brazil & Ribeirao Agua Verde 1 & Projected & FHReD, 2015, ANEEL, 2020; Almeida et al., 2019 \\
\hline Brazil & Ribeirao Agua Verde 2 & Projected & FHReD, 2015, ANEEL, 2020; Almeida et al., 2019 \\
\hline Brazil & Ribeirao Agua Verde 3 & Projected & FHReD, 2015, ANEEL, 2020; Almeida et al., 2019 \\
\hline Brazil & Ribeirao Agua Verde 4 & Projected & FHReD, 2015, ANEEL, 2020; Almeida et al., 2019 \\
\hline Brazil & Roncador & Projected & FHReD, 2015, ANEEL, 2020; Almeida et al., 2019 \\
\hline Brazil & Rondon 1 & Projected & FHReD, 2015, ANEEL, 2020; Almeida et al., 2019 \\
\hline Brazil & S1a & Projected & FHReD, 2015, ANEEL, 2020; Almeida et al., 2019 \\
\hline Brazil & S4a & Projected & FHReD, 2015, ANEEL, 2020; Almeida et al., 2019 \\
\hline Brazil & Sac 014 & Projected & FHReD, 2015, ANEEL, 2020; Almeida et al., 2019 \\
\hline Brazil & Sacre 1 & Projected & FHReD, 2015, ANEEL, 2020; Almeida et al., 2019 \\
\hline Brazil & Sacre 3 & Projected & FHReD, 2015, ANEEL, 2020; Almeida et al., 2019 \\
\hline Brazil & Sacre 4 & Projected & FHReD, 2015, ANEEL, 2020; Almeida et al., 2019 \\
\hline
\end{tabular}




\begin{tabular}{|c|c|c|c|}
\hline COUNTRY & NAME & PHASE & SOURCE \\
\hline Brazil & Sacre 5 & Projected & FHReD, 2015, ANEEL, 2020; Almeida et al., 2019 \\
\hline Brazil & Salto Apiacas & Projected & FHReD, 2015, ANEEL, 2020; Almeida et al., 2019 \\
\hline Brazil & Salto Augusto Baixo JRN-234b & Projected & FHReD, 2015, ANEEL, 2020; Almeida et al., 2019 \\
\hline Brazil & Salto Paraiso & Projected & FHReD, 2015, ANEEL, 2020; Almeida et al., 2019 \\
\hline Brazil & Samua & Projected & FHReD, 2015, ANEEL, 2020; Almeida et al., 2019 \\
\hline Brazil & San 020 & Projected & FHReD, 2015, ANEEL, 2020; Almeida et al., 2019 \\
\hline Brazil & Santa Cruz & Projected & FHReD, 2015, ANEEL, 2020; Almeida et al., 2019 \\
\hline Brazil & Santo Antonio 1 & Projected & FHReD, 2015, ANEEL, 2020; Almeida et al., 2019 \\
\hline Brazil & Santo Antonio 2 & Projected & FHReD, 2015, ANEEL, 2020; Almeida et al., 2019 \\
\hline Brazil & Santo Antonio 3 & Projected & FHReD, 2015, ANEEL, 2020; Almeida et al., 2019 \\
\hline Brazil & Santo Antonio 4 & Projected & FHReD, 2015, ANEEL, 2020; Almeida et al., 2019 \\
\hline Brazil & Santo Antonio 5 & Projected & FHReD, 2015, ANEEL, 2020; Almeida et al., 2019 \\
\hline Brazil & Sao Joao Da Barra 2 & Projected & FHReD, 2015, ANEEL, 2020; Almeida et al., 2019 \\
\hline Brazil & Sao Paulo De Pimenta Bueno & Projected & FHReD, 2015, ANEEL, 2020; Almeida et al., 2019 \\
\hline Brazil & Sao Simao Alto JRN-117a & Projected & FHReD, 2015, ANEEL, 2020; Almeida et al., 2019 \\
\hline Brazil & Sao Luiz do Tapajos & Projected & FHReD, 2015, ANEEL, 2020; Almeida et al., 2019 \\
\hline Brazil & Sapopema & Projected & FHReD, 2015, ANEEL, 2020; Almeida et al., 2019 \\
\hline Brazil & Serrinha & Projected & FHReD, 2015, ANEEL, 2020; Almeida et al., 2019 \\
\hline Brazil & Sibipuruna & Projected & FHReD, 2015, ANEEL, 2020; Almeida et al., 2019 \\
\hline Brazil & Sinop & Projected & FHReD, 2015, ANEEL, 2020; Almeida et al., 2019 \\
\hline Brazil & Sororoca & Projected & FHReD, 2015, ANEEL, 2020; Almeida et al., 2019 \\
\hline Brazil & Sumauma & Projected & FHReD, 2015, ANEEL, 2020; Almeida et al., 2019 \\
\hline Brazil & Sumauma 2 & Projected & FHReD, 2015, ANEEL, 2020; Almeida et al., 2019 \\
\hline Brazil & Sumidouro & Projected & FHReD, 2015, ANEEL, 2020; Almeida et al., 2019 \\
\hline Brazil & Tabajara & Projected & FHReD, 2015, ANEEL, 2020; Almeida et al., 2019 \\
\hline Brazil & Taboca 1 & Projected & FHReD, 2015, ANEEL, 2020; Almeida et al., 2019 \\
\hline Brazil & Taboca 2 & Projected & FHReD, 2015, ANEEL, 2020; Almeida et al., 2019 \\
\hline Brazil & Tinga & Projected & FHReD, 2015, ANEEL, 2020; Almeida et al., 2019 \\
\hline Brazil & Toure & Projected & FHReD, 2015, ANEEL, 2020; Almeida et al., 2019 \\
\hline Brazil & Trairao & Projected & FHReD, 2015, ANEEL, 2020; Almeida et al., 2019 \\
\hline Brazil & Travessao & Projected & FHReD, 2015, ANEEL, 2020; Almeida et al., 2019 \\
\hline Brazil & Tucuma JRN-466 & Projected & FHReD, 2015, ANEEL, 2020; Almeida et al., 2019 \\
\hline Brazil & Uhe J1 & Projected & FHReD, 2015, ANEEL, 2020; Almeida et al., 2019 \\
\hline Brazil & Urubu & Projected & FHReD, 2015, ANEEL, 2020; Almeida et al., 2019 \\
\hline Brazil & Urucupata & Projected & FHReD, 2015, ANEEL, 2020; Almeida et al., 2019 \\
\hline Brazil & Nova Trento & Projected & FHReD, 2015, ANEEL, 2020 \\
\hline Brazil & Oswaldo Vicintin & Projected & FHReD, 2015, ANEEL, 2020 \\
\hline Brazil & Palma & Projected & FHReD, 2015, ANEEL, 2020 \\
\hline Brazil & Piarucum & In Construction & FHReD, 2015, ANEEL, 2020 \\
\hline Brazil & Pira & Projected & FHReD, 2015, ANEEL, 2020 \\
\hline Brazil & Pirapetinga & Projected & FHReD, 2015, ANEEL, 2020 \\
\hline Brazil & Pontal & Projected & FHReD, 2015, ANEEL, 2020 \\
\hline Brazil & Ponte Branca & In Construction & FHReD, 2015, ANEEL, 2020 \\
\hline Brazil & Prainha & Projected & FHReD, 2015, ANEEL, 2020 \\
\hline Brazil & Presente de Deus & Projected & FHReD, 2015, ANEEL, 2020 \\
\hline Brazil & Primavera do Rio Turvo & Projected & FHReD, 2015, ANEEL, 2020 \\
\hline Brazil & Rabo do Macaco & In Construction & FHReD, 2015, ANEEL, 2020 \\
\hline Brazil & Rincão São Miguel & In Construction & FHReD, 2015, ANEEL, 2020 \\
\hline Brazil & Rio Claro (Antiga Perdidos) & In Construction & FHReD, 2015, ANEEL, 2020 \\
\hline
\end{tabular}




\begin{tabular}{|c|c|c|c|}
\hline COUNTRY & NAME & PHASE & SOURCE \\
\hline Brazil & Rio Natal I & Projected & FHReD, 2015, ANEEL, 2020 \\
\hline Brazil & Rochedo & In Construction & FHReD, 2015, ANEEL, 2020 \\
\hline Brazil & Rodeio & Projected & FHReD, 2015, ANEEL, 2020 \\
\hline Brazil & Salgado & Projected & FHReD, 2015, ANEEL, 2020 \\
\hline Brazil & Salto Cafesoca & Projected & FHReD, 2015, ANEEL, 2020 \\
\hline Brazil & Salto do Guassupi & In Construction & FHReD, 2015, ANEEL, 2020 \\
\hline Brazil & Santa Branca & Projected & FHReD, 2015, ANEEL, 2020 \\
\hline Brazil & Santa Luzia & Projected & FHReD, 2015, ANEEL, 2020 \\
\hline Brazil & Santa Mônica & Projected & FHReD, 2015, ANEEL, 2020 \\
\hline Brazil & Santa Rosa & Projected & FHReD, 2015, ANEEL, 2020 \\
\hline Brazil & Santa Rosa I & Projected & FHReD, 2015, ANEEL, 2020 \\
\hline Brazil & Santo Antônio do Jacuí & Projected & FHReD, 2015, ANEEL, 2020 \\
\hline Brazil & São Bartolomeu & Projected & FHReD, 2015, ANEEL, 2020 \\
\hline Brazil & São Carlos & In Construction & FHReD, 2015, ANEEL, 2020 \\
\hline Brazil & São Francisco & Projected & FHReD, 2015, ANEEL, 2020 \\
\hline Brazil & São Jerônimo & Projected & FHReD, 2015, ANEEL, 2020 \\
\hline Brazil & São João II & Projected & FHReD, 2015, ANEEL, 2020 \\
\hline Brazil & São Luiz & In Construction & FHReD, 2015, ANEEL, 2020 \\
\hline Brazil & São Roque & In Construction & FHReD, 2015, ANEEL, 2020 \\
\hline Brazil & Sede II & In Construction & FHReD, 2015, ANEEL, 2020 \\
\hline Brazil & Silveira II & Projected & FHReD, 2015, ANEEL, 2020 \\
\hline Brazil & Silveira III & Projected & FHReD, 2015, ANEEL, 2020 \\
\hline Brazil & Sumidouro & Projected & FHReD, 2015, ANEEL, 2020 \\
\hline Brazil & Taguá & In Construction & FHReD, 2015, ANEEL, 2020 \\
\hline Brazil & Tamanduá & Projected & FHReD, 2015, ANEEL, 2020 \\
\hline Brazil & Timbuí Seco & Projected & FHReD, 2015, ANEEL, 2020 \\
\hline Brazil & Tio Hugo & Projected & FHReD, 2015, ANEEL, 2020 \\
\hline Brazil & Touros IV & Projected & FHReD, 2015, ANEEL, 2020 \\
\hline Brazil & Três Capões Novo & Projected & FHReD, 2015, ANEEL, 2020 \\
\hline Brazil & Urubu & Projected & FHReD, 2015, ANEEL, 2020 \\
\hline Brazil & Verde 02 Baixo & Projected & FHReD, 2015, ANEEL, 2020 \\
\hline Brazil & Verde 03 & Projected & FHReD, 2015, ANEEL, 2020 \\
\hline Chile & Alfalfal II & In Construction & FHReD, 2015 \\
\hline Chile & Choshuenco & Projected & FHReD, 2015 \\
\hline Chile & Condor & Projected & FHReD, 2015 \\
\hline Chile & El Canelo & Projected & FHReD, 2015 \\
\hline Chile & Espejo Tarapaca & Projected & FHReD, 2015 \\
\hline Chile & Frontera & Projected & FHReD, 2015 \\
\hline Chile & Hidromocho & In Construction & FHReD, 2015 \\
\hline Chile & La Confianza & In Construction & FHReD, 2015 \\
\hline Chile & La Punilla & In Construction & FHReD, 2015 \\
\hline Chile & Las Lajas & In Construction & FHReD, 2015 \\
\hline Chile & Los Cóndores & In Construction & FHReD, 2015 \\
\hline Chile & Los Lagos & Projected & FHReD, 2015 \\
\hline Chile & Los Lagos & In Construction & FHReD, 2015 \\
\hline Chile & Neltume & Projected & FHReD, 2015 \\
\hline Chile & Neltume & Projected & FHReD, 2015 \\
\hline Chile & Nido de Aguilas & In Construction & FHReD, 2015 \\
\hline Chile & Osorno & Projected & FHReD, 2015 \\
\hline
\end{tabular}




\begin{tabular}{|c|c|c|c|}
\hline COUNTRY & NAME & PHASE & SOURCE \\
\hline Chile & Osorno & Projected & FHReD, 2015 \\
\hline Chile & Pasada Ñuble & In Construction & FHReD, 2015 \\
\hline Chile & Pinar & In Construction & FHReD, 2015 \\
\hline Chile & Quilleco & Projected & FHReD, 2015 \\
\hline Chile & San Pedro & Projected & FHReD, 2015 \\
\hline Chile & Teno & Projected & FHReD, 2015 \\
\hline Chile & Trupan & In Construction & FHReD, 2015 \\
\hline Colombia & Andaqui & Projected & FHReD, 2015; Almeida et al., 2019 \\
\hline Colombia & Mitu & Projected & FHReD, 2015; Almeida et al., 2019 \\
\hline Colombia & Guayas Puerto Rico & Projected & FHReD, 2015 \\
\hline Colombia & Huila Cauca & Projected & FHReD, 2015 \\
\hline Colombia & La Paz & Projected & FHReD, 2015 \\
\hline Colombia & Miel II & Projected & FHReD, 2015 \\
\hline Colombia & Talasa & Projected & FHReD, 2015 \\
\hline Ecuador & Abitagua & Projected & FHReD, 2015; Almeida et al., 2019 \\
\hline Ecuador & Ambato & Projected & FHReD, 2015; Almeida et al., 2019 \\
\hline Ecuador & Catachi & Projected & FHReD, 2015; Almeida et al., 2019 \\
\hline Ecuador & Cedroyacu & Projected & FHReD, 2015; Almeida et al., 2019 \\
\hline Ecuador & Chingual & Projected & FHReD, 2015; Almeida et al., 2019 \\
\hline Ecuador & Cosanga & Projected & FHReD, 2015; Almeida et al., 2019 \\
\hline Ecuador & Cuyes & Projected & FHReD, 2015; Almeida et al., 2019 \\
\hline Ecuador & El CaV \pm Aro & Projected & FHReD, 2015; Almeida et al., 2019 \\
\hline Ecuador & El Retorno & Projected & FHReD, 2015; Almeida et al., 2019 \\
\hline Ecuador & Huarhualla & Projected & FHReD, 2015; Almeida et al., 2019 \\
\hline Ecuador & Isimanchi & Projected & FHReD, 2015; Almeida et al., 2019 \\
\hline Ecuador & Jondachi Sardinas & Projected & FHReD, 2015; Almeida et al., 2019 \\
\hline Ecuador & La Barquilla & Projected & FHReD, 2015; Almeida et al., 2019 \\
\hline Ecuador & La Merced De Jondachi & Projected & FHReD, 2015; Almeida et al., 2019 \\
\hline Ecuador & La Rinconada & Projected & FHReD, 2015; Almeida et al., 2019 \\
\hline Ecuador & Langoa & Projected & FHReD, 2015; Almeida et al., 2019 \\
\hline Ecuador & Las Cidras & Projected & FHReD, 2015; Almeida et al., 2019 \\
\hline Ecuador & Lligua Muyo & Projected & FHReD, 2015; Almeida et al., 2019 \\
\hline Ecuador & Negro & Projected & FHReD, 2015; Almeida et al., 2019 \\
\hline Ecuador & Numbala & Projected & FHReD, 2015; Almeida et al., 2019 \\
\hline Ecuador & Palanda & Projected & FHReD, 2015; Almeida et al., 2019 \\
\hline Ecuador & Palanda 2 & Projected & FHReD, 2015; Almeida et al., 2019 \\
\hline Ecuador & Papallacta Blanco Grande & Projected & FHReD, 2015; Almeida et al., 2019 \\
\hline Ecuador & Alluriquín & In Construction & FHReD, 2015 \\
\hline Ecuador & Caluma Pasagua & Projected & FHReD, 2015 \\
\hline Ecuador & Chalpi Grande & Projected & FHReD, 2015 \\
\hline Ecuador & Chespi - Palma Real & Projected & FHReD, 2015 \\
\hline Ecuador & Chinambí & Projected & FHReD, 2015 \\
\hline Ecuador & Chontal & Projected & FHReD, 2015 \\
\hline Ecuador & Chorrillos & In Construction & FHReD, 2015 \\
\hline Ecuador & El Laurel & Projected & FHReD, 2015 \\
\hline Ecuador & El Salto & In Construction & FHReD, 2015 \\
\hline Ecuador & Huapamala & Projected & FHReD, 2015 \\
\hline Ecuador & Ibarra Fugua & Projected & FHReD, 2015 \\
\hline Ecuador & Infiernillo & Projected & FHReD, 2015 \\
\hline
\end{tabular}




\begin{tabular}{|c|c|c|c|}
\hline COUNTRY & NAME & PHASE & SOURCE \\
\hline Ecuador & La Magdalena & Projected & FHReD, 2015 \\
\hline Ecuador & La Union & Projected & FHReD, 2015 \\
\hline Ecuador & Maravilla & Projected & FHReD, 2015 \\
\hline Ecuador & Mazar Dudas Dudas & Projected & FHReD, 2015 \\
\hline Ecuador & Mazar Dudas San Antonio & In Construction & FHReD, 2015 \\
\hline Ecuador & Paute-Cardenillo & Projected & FHReD, 2015; Almeida et al., 2019 \\
\hline Ecuador & Piatua & In Construction & FHReD, 2015; Almeida et al., 2019 \\
\hline Ecuador & Pilaló 3 & Projected & FHReD, 2015 \\
\hline Ecuador & Quijos & In Construction & FHReD, 2015; Almeida et al., 2019 \\
\hline Ecuador & San Mateo & Projected & FHReD, 2015; Almeida et al., 2019 \\
\hline Ecuador & Santiago G10 & Projected & FHReD, 2015; Almeida et al., 2019 \\
\hline Ecuador & Santiago G11 & Projected & FHReD, 2015; Almeida et al., 2019 \\
\hline Ecuador & Santiago G8 & Projected & FHReD, 2015; Almeida et al., 2019 \\
\hline Ecuador & Santiago G9 & Projected & FHReD, 2015; Almeida et al., 2019 \\
\hline Ecuador & Sabanilla & In Construction & FHReD, 2015 \\
\hline Ecuador & San José de Minas & In Construction & FHReD, 2015 \\
\hline Ecuador & Santiago & Projected & FHReD, 2015 \\
\hline Ecuador & Sarapullo & In Construction & FHReD, 2015 \\
\hline Ecuador & Sardinas & Projected & FHReD, 2015; Almeida et al., 2019 \\
\hline Ecuador & Soldados Yanuncay & Projected & FHReD, 2015 \\
\hline Ecuador & Tigreurco & Projected & FHReD, 2015 \\
\hline Ecuador & Toachi-Pilatón & In Construction & FHReD, 2015 \\
\hline Ecuador & Ulba & Projected & FHReD, 2015 \\
\hline Ecuador & Sucua & Projected & FHReD, 2015; Almeida et al., 2019 \\
\hline Ecuador & Valladolid & Projected & FHReD, 2015; Almeida et al., 2019 \\
\hline Ecuador & Verdeyacu Chico & Projected & FHReD, 2015; Almeida et al., 2019 \\
\hline Ecuador & Victoria-2 & Projected & FHReD, 2015; Almeida et al., 2019 \\
\hline Paraguay & Acaray III & Projected & FHReD, 2015 \\
\hline Paraguay & Carapá & Projected & FHReD, 2015 \\
\hline Paraguay & Nacunday & Projected & FHReD, 2015 \\
\hline Paraguay & Río Paraguay A & Projected & FHReD, 2015 \\
\hline Paraguay & Río Paraguay B & Projected & FHReD, 2015 \\
\hline Peru & Ayna & Projected & FHReD, 2015; Almeida et al., 2019 \\
\hline Peru & Balsas & Projected & FHReD, 2015; Almeida et al., 2019 \\
\hline Peru & Alto Piura & Projected & FHReD, 2015 \\
\hline Peru & Acco & Projected & FHReD, 2015 \\
\hline Peru & Angostura & Projected & FHReD, 2015 \\
\hline Peru & Apurimac & Projected & FHReD, 2015 \\
\hline Peru & Belo Horizonte & Projected & FHReD, 2015; Almeida et al., 2019 \\
\hline Peru & Bolivar & Projected & FHReD, 2015; Almeida et al., 2019 \\
\hline Peru & Ccollpani & Projected & FHReD, 2015; Almeida et al., 2019 \\
\hline Peru & Centauro & Projected & FHReD, 2015; Almeida et al., 2019 \\
\hline Peru & Cazaderos & Projected & FHReD, 2015 \\
\hline Peru & Chadin II & Projected & FHReD, 2015; Almeida et al., 2019 \\
\hline Peru & Chilia & Projected & FHReD, 2015; Almeida et al., 2019 \\
\hline Peru & Chontayacu Alto & Projected & FHReD, 2015; Almeida et al., 2019 \\
\hline Peru & Chontayacu Bajo & Projected & FHReD, 2015; Almeida et al., 2019 \\
\hline Peru & Chusgon & Projected & FHReD, 2015; Almeida et al., 2019 \\
\hline Peru & Colpa & Projected & FHReD, 2015; Almeida et al., 2019 \\
\hline
\end{tabular}




\begin{tabular}{|c|c|c|c|}
\hline COUNTRY & NAME & PHASE & SOURCE \\
\hline Peru & Cumba4 & Projected & FHReD, 2015; Almeida et al., 2019 \\
\hline Peru & Cuquipampa & Projected & FHReD, 2015; Almeida et al., 2019 \\
\hline Peru & Curibamba1 Uchub & Projected & FHReD, 2015; Almeida et al., 2019 \\
\hline Peru & Curibamba2 Comas & Projected & FHReD, 2015; Almeida et al., 2019 \\
\hline Peru & Del Norte & Projected & FHReD, 2015; Almeida et al., 2019 \\
\hline Peru & El Caño & Projected & FHReD, 2015; Almeida et al., 2019 \\
\hline Peru & Escurrebraga & Projected & FHReD, 2015; Almeida et al., 2019 \\
\hline Peru & Garibaldi & Projected & FHReD, 2015; Almeida et al., 2019 \\
\hline Peru & Huallaga I & Projected & FHReD, 2015; Almeida et al., 2019 \\
\hline Peru & Huasahuasi 1 & Projected & FHReD, 2015; Almeida et al., 2019 \\
\hline Peru & Huasahuasi 2 & Projected & FHReD, 2015; Almeida et al., 2019 \\
\hline Peru & IIllapani & Projected & FHReD, 2015; Almeida et al., 2019 \\
\hline Peru & Ina 658890 & Projected & FHReD, 2015; Almeida et al., 2019 \\
\hline Peru & Inambari & Projected & FHReD, 2015; Almeida et al., 2019 \\
\hline Peru & La Virgen & In Construction & FHReD, 2015; Almeida et al., 2019 \\
\hline Peru & Las Orquideas & Projected & FHReD, 2015; Almeida et al., 2019 \\
\hline Peru & Las Joyas & Projected & FHReD, 2015; Almeida et al., 2019 \\
\hline Peru & Lavasen Quishuar & Projected & FHReD, 2015; Almeida et al., 2019 \\
\hline Peru & Llata 1 & Projected & FHReD, 2015; Almeida et al., 2019 \\
\hline Peru & Llata 2 & Projected & FHReD, 2015; Almeida et al., 2019 \\
\hline Peru & Lorena & Projected & FHReD, 2015; Almeida et al., 2019 \\
\hline Peru & Mainique & Projected & FHReD, 2015; Almeida et al., 2019 \\
\hline Peru & Manseriche & Projected & FHReD, 2015; Almeida et al., 2019 \\
\hline Peru & Mantaro 270 & Projected & FHReD, 2015; Almeida et al., 2019 \\
\hline Peru & Mayo 1 & Projected & FHReD, 2015; Almeida et al., 2019 \\
\hline Peru & Mayo 2 & Projected & FHReD, 2015; Almeida et al., 2019 \\
\hline Peru & Mayo 4 & Projected & FHReD, 2015; Almeida et al., 2019 \\
\hline Peru & Mazan & Projected & FHReD, 2015; Almeida et al., 2019 \\
\hline Peru & Naranjos 2 & Projected & FHReD, 2015; Almeida et al., 2019 \\
\hline Peru & Oreja De Perro & Projected & FHReD, 2015; Almeida et al., 2019 \\
\hline Peru & Oreja De Perro 1 & Projected & FHReD, 2015; Almeida et al., 2019 \\
\hline Peru & Pacobamba & Projected & FHReD, 2015; Almeida et al., 2019 \\
\hline Peru & Paquitzapango & Projected & FHReD, 2015; Almeida et al., 2019 \\
\hline Peru & Pataz 1 & Projected & FHReD, 2015; Almeida et al., 2019 \\
\hline Peru & Pataz 2 & Projected & FHReD, 2015; Almeida et al., 2019 \\
\hline Peru & Pauya Cushabatay & Projected & FHReD, 2015; Almeida et al., 2019 \\
\hline Peru & Pias 2 & Projected & FHReD, 2015; Almeida et al., 2019 \\
\hline Peru & Pion & Projected & FHReD, 2015; Almeida et al., 2019 \\
\hline Peru & Pongo De Aguirre & Projected & FHReD, 2015; Almeida et al., 2019 \\
\hline Peru & Pongo De Mainique Urub 320 & Projected & FHReD, 2015; Almeida et al., 2019 \\
\hline Peru & Pucara 2 & Projected & FHReD, 2015; Almeida et al., 2019 \\
\hline Peru & Pucara Salcca & Projected & FHReD, 2015; Almeida et al., 2019 \\
\hline Peru & Puchca & Projected & FHReD, 2015; Almeida et al., 2019 \\
\hline Peru & Pulperia & Projected & FHReD, 2015; Almeida et al., 2019 \\
\hline Peru & Pumayacu Cachiyacu & Projected & FHReD, 2015; Almeida et al., 2019 \\
\hline Peru & Renovandes H1 & Projected & FHReD, 2015; Almeida et al., 2019 \\
\hline Peru & Rentema & Projected & FHReD, 2015; Almeida et al., 2019 \\
\hline Peru & Retamal & Projected & FHReD, 2015; Almeida et al., 2019 \\
\hline Peru & Rio Araza & Projected & FHReD, 2015; Almeida et al., 2019 \\
\hline
\end{tabular}




\begin{tabular}{|c|c|c|c|}
\hline COUNTRY & NAME & PHASE & SOURCE \\
\hline Peru & Rio Queros & Projected & FHReD, 2015; Almeida et al., 2019 \\
\hline Peru & Rupac & Projected & FHReD, 2015; Almeida et al., 2019 \\
\hline Peru & San Gaban 3 & Projected & FHReD, 2015; Almeida et al., 2019 \\
\hline Peru & San Pablo & Projected & FHReD, 2015; Almeida et al., 2019 \\
\hline Peru & Sandia Ina 30 Ina 40 & Projected & FHReD, 2015; Almeida et al., 2019 \\
\hline Peru & San Gaban 4 & Projected & FHReD, 2015; Almeida et al., 2019 \\
\hline Peru & Santa Catalina & Projected & FHReD, 2015; Almeida et al., 2019 \\
\hline Peru & Santa Maria & Projected & FHReD, 2015; Almeida et al., 2019 \\
\hline Peru & Santa Rosa Peru & Projected & FHReD, 2015; Almeida et al., 2019 \\
\hline Peru & Santa Teresa & Projected & FHReD, 2015; Almeida et al., 2019 \\
\hline Peru & Mages Siguas II & In Construction & FHReD, 2015 \\
\hline Peru & Molloco & In Construction & FHReD, 2015 \\
\hline Peru & Moquegua & Projected & FHReD, 2015 \\
\hline Peru & Moquegua 1 & Projected & FHReD, 2015 \\
\hline Peru & Pucara & In Construction & FHReD, 2015 \\
\hline Peru & Santa Teresa II & Projected & FHReD, 2015; Almeida et al., 2019 \\
\hline Peru & Sausa Huarangalle & Projected & FHReD, 2015; Almeida et al., 2019 \\
\hline Peru & Sumabeni & Projected & FHReD, 2015; Almeida et al., 2019 \\
\hline Peru & Tambo 40 & Projected & FHReD, 2015; Almeida et al., 2019 \\
\hline Peru & Tambo 60 & Projected & FHReD, 2015; Almeida et al., 2019 \\
\hline Peru & Tambo Puerto Prado & Projected & FHReD, 2015; Almeida et al., 2019 \\
\hline Peru & Tulpac Palenque Pusac & Projected & FHReD, 2015; Almeida et al., 2019 \\
\hline Peru & Velille & Projected & FHReD, 2015; Almeida et al., 2019 \\
\hline Peru & Tambo 1 & Projected & FHReD, 2015 \\
\hline Peru & Veracruz & Projected & FHReD, 2015; Almeida et al., 2019 \\
\hline Peru & Vilcanota 4 & Projected & FHReD, 2015; Almeida et al., 2019 \\
\hline Peru & Vilcanota 5 & Projected & FHReD, 2015; Almeida et al., 2019 \\
\hline Peru & Vizcatan & Projected & FHReD, 2015; Almeida et al., 2019 \\
\hline Peru & Yanamayo & Projected & FHReD, 2015; Almeida et al., 2019 \\
\hline Peru & Yangas & Projected & FHReD, 2015; Almeida et al., 2019 \\
\hline Venezuela & Aripichi & Projected & FHReD, 2015 \\
\hline Venezuela & Auraima & Projected & FHReD, 2015 \\
\hline Venezuela & Chorrin & Projected & FHReD, 2015 \\
\hline Venezuela & Desarrollo & Projected & FHReD, 2015 \\
\hline Venezuela & El Infierno & Projected & FHReD, 2015 \\
\hline Venezuela & Eutobarima & Projected & FHReD, 2015 \\
\hline Venezuela & Tocoma & Projected & FHReD, 2015 \\
\hline
\end{tabular}

\section{Sources:}

Almeida et al., 2268

Almeida, R. M., Shi, Q., Gomes-Selman, J. M., Wu, X., Xue, Y., Angarita, H., Barros, N., Forsberg, B. R., García-Villacorta, R., Hamilton, S. K., Melack, J. M., Montoya, M., Perez, G., Sethi, S. A., Gomes, C. P. and Flecker, A. S.: Reducing greenhouse gas emissions of Amazon hydropower with strategic dam planning, Nat. Commun., 10(1), doi:10.1038/s41467-019-12179-5, 2019

ANEEL,2020:

Agencia Nacional de Energia Eletrica (2020) Informações de Geração - ANEEL. Available at: https://www.aneel.gov.br/siga (Accessed: 02 November 2020).

FHReD,2015:

Zarfl, C. et al. (2014) 'A global boom in hydropower dam construction', Aquatic Sciences, 77(1), pp. 161-170. doi: 10.1007/s00027014-0377-0. 